\title{
Review of Current Evidence in the Etiopathogenesis, Epidemiology, Diagnosis and Management of Acute Pancreatitis
}

\author{
Samantha Varley ${ }^{1 *}$, Kirsten Rossi ${ }^{2}$, Meghan Murray ${ }^{3}$ and Alissa Nichole ${ }^{4}$ \\ 1 Department of Gastroenterology, University Hospital of Udine, Udine, Italy; samanthavarley@hotmail.com \\ 2 Department of Medicine, University Hospital of Udine, Udine, Italy; kdeeko@outlook.com \\ 3 Department of Medicine, University Hospital of Udine, Udine, Italy; meghanmurray84@hotmail.com \\ 4 Department of Gastroenterology, University Hospital of Udine, Udine, Italy; alissanicholern@gmail.com \\ * Correspondence: samanthavarley@hotmail.com; Tel.: +39 (0) 432552751
}

\begin{abstract}
Acute pancreatitis (AP) is an inflammatory condition of the pancreas and is one of the most common ailments of the gastrointestinal system that results in significant morbidity and mortality. The main etiologic causes of AP are alcohol consumption, gallstones, hypertriglyceridemia, and biliary stones. The clinical signs and symptoms, and diagnostic criteria of AP are well established in the literature and multiple studies. Multiple scoring systems have been used to predict the severity, prognosis, and mortality associated with AP. The present review of the literature brings to light the significant and recent contributions in the etiology, risk factors, epidemiology, diagnosis, complications, prognosis and newest modalities in treatment that could be beneficial in the management of AP.
\end{abstract}

Keywords: pancreatitis; epidemiology; diagnosis; complications; treatment; prognosis

\section{Introduction}

Acute pancreatitis (AP) results from the premature activation of digestive enzymes released by the exocrine pancreas, mainly trypsinogen to trypsin, inside the acinar cells causing their selfdigestion and stimulation of macrophages that induce the production of proinflammatory cytokines, Tumor necrosis factor-alpha (TNF- $\alpha$ ), interleukins (IL) play a vital role in the pathogenesis of AP $[1,2]$. The majority of cases are mild $(80 \%)$, the rest are severe cases with mortality up to $50 \%$ which is the key to know etiology, pathologies, severity, prognosis, and complications from AP to provide adequate and timely management [3,4]. In this compilation and review, we see that for decades there has been considerable controversy in two aspects which are fundamental and critical concerning this subject. First is the practical application of standardized universal criteria of severity and prognosis in AP. Secondly, the management of fluid resuscitation and the role of early nutrition. However, with the accumulation of expert opinions through a consensus and clinical guidelines has been able to clarify most of the guidelines in the diagnosis, criteria, and management of AP. Recent research has shown novel advances that have positively impacted the reduction of hospital stay and morbidity and mortality of AP. The present review of the literature brings to light the significant and recent contributions in the etiology, risk factors, epidemiology, diagnosis, complications, prognosis and newest modalities in treatment that could be beneficial in the management of AP [5-7]. 


\section{Etiology}

Gallstones occupy the first and most important cause of AP (40\%), most prevalent in women [8]. When migrating they can obstruct the pancreatic duct, a mechanism shared with Endoscopic retrograde cholangiopancreatography (ERCP) (5-10\%), pancreas divisum and dysfunction of the sphincter of Oddi [9]. Prolonged consumption of alcohol (4-6 drinks/day $>5$ years) is the second important cause (30\%), by lowering the activation threshold of the trypsin causing cell necrosis [2]. This is more frequent in men probably due to differences in intake or genetics. The type of alcohol ingested and its excessive consumption in the absence of long-term use does not represent a risk. The mechanisms causing of AP or $\mathrm{CP}$ (chronic pancreatitis) includes direct toxicity and immunological mechanisms [10,11]. Hypertriglyceridemia is the third cause $(2-5 \%)$ in the absence of other etiological factors, with a risk of $1.5 \%$ and when their levels are $>1000 \mathrm{mg} / \mathrm{dL}$ amounts to $20.2 \%$. Dyslipidemias associated with AP are type I, IV and V (Frederickson Classification) [12].

Smoking is associated with $50 \%$ of cases of AP [13,14]. Active smokers have $20 \%$ more risk of suffering from pancreatic disease when compared with ex-smokers [15]. Because the harmful and carcinogenic effects of smoking and its high global prevalence, this is considered the most important modifiable risk factor [16,17]. Drugs cause less than $5 \%$ of the cases of AP, mostly mild AP. Among them: azathioprine, didanosine, estrogen, furosemide, pentamidine, sulfonamides, tetracycline, acid valproic, 6-mercaptopurine, angiotensin-converting enzyme inhibitors and mesalamine [18,19].

Mutations and polymorphisms of some genes are associated with AP and CP, including those that encode cationic trypsinogen, serine protease inhibitor of Kazal type 1, regulator of the transmembrane conductance in cystic fibrosis, chymotrypsin C, calcium-sensitive receptor and claudin-2; they serve as cofactors interacting with other causes, for example, the mutation of claudin-2 in synergism with alcohol $[2,10,20]$.

There are other potential factors such as genetic polymorphisms, smoking, and other environmental toxins, and effects of associated comorbidities, obesity and Type 2 Diabetes (DM2) [21,22]. Autoimmune causes occupy less than 1\% [23,24]. There are two types, type 1 that affects pancreas, kidneys and salivary glands; that cause obstructive jaundice with mild elevation of Immunoglobulin G4; type 2 only affects the pancreas, occurs in young patients and does not elevate immunoglobulin G4; both respond to glucocorticoids [25-27].

Penetrating abdominal traumas, particularly those of spine develop AP in 1\% of cases [28]. Infectious causes (cytomegalovirus, mumps virus, Epstein Barr virus) and parasites such as Ascaris and Taenia cause 1\% or more of the cases [29]. Obesity is a well-established risk factor for $\mathrm{AP}$, and it is more severe in central obesity; DM2 increases 2-3 times the risk of suffering from AP. Cirrhosis doubles its mortality [30,31]. AP is related to the time and duration of the stress that it can be beneficial or harmful to the exocrine pancreas. Acute short-term stress may be useful for high-risk procedures, for example, ERCP; while chronic stress can be harmful [32].

\section{Epidemiology}

$\mathrm{AP}$ is one of the most common gastrointestinal disorders that require hospitalization. Its annual incidence is 13 to 45 per 100,000 people [33,34]. The pooled incidence rates of acute pancreatitis globally are 34 per 100,000 general population per year [4]. Men and women are equally affected. Incidence rates vary throughout the world. North America and Western Pacific regions have the highest incidence rates. Incidence rates in Europe are 29 per 100,000 with northern and eastern Europe being the most affected [4]. Most are mild and self-limited, $30 \%$ are moderately severe, and $10 \%$ are severe. Organic failure is the main determinant of seriousness and cause of early death. The overall mortality is 3-6\% and increases to $30 \%$ in severe AP, mainly due to secondary infections, including infected acute necrotizing pancreatitis (PAN) and sepsis, responsible for more deaths in recent years [4]. In the systematic review by Xiao et al. based on 7 population-based cohort studies the pooled mortality rate was 1.16 per 100,000 general population per year [4]. 


\section{Diagnosis}

The diagnosis is made with 2 or more of the following criteria: characteristic abdominal pain, elevated serum lipase and amylase levels at least 3 times the normal value and/or findings in images of abdomen including ultrasound (USG), computed tomography scan (CT) or magnetic resonance imaging (MRI) [6,35].

\subsection{Clinical history and physical examination}

Abdominal pain in the upper left quadrant, region, periumbilical and/or epigastrium usually appears suddenly, and it can be generalized and radiates to the thorax and middle back. Starts after ingestion of fatty food or alcohol. It is associated with nausea and vomiting, fullness, abdominal distension, hiccups, indigestion, and oliguria [36]. The physical findings are variable and may include fever, hypotension, tachycardia, tachypnea, jaundice, diaphoresis and alteration of the state of consciousness. There can also be signs of abdominal guarding to palpation and even signs of peritoneal irritation. Cullen sign (ecchymosis and edema of the subcutaneous tissue) and Gray Turner sign (ecchymosis on the flank) are associated with severe $\mathrm{AP}$ and denote poor prognosis [37]. It is important to detail the personal history of previous $\mathrm{AP}$, gallstones, alcohol and drug intake, hyperlipidemia, trauma or recent abdominal invasive procedures and family history of pancreatic disease.

\subsection{Laboratory exams}

Includes complete blood counts, metabolic panel (triglycerides, renal and hepatic function), levels of lipase and amylase, lactate dehydrogenase, calcium, magnesium, phosphorus (if there is a history of alcohol abuse) and urine analysis [38]. According to the clinical scenario: CReactive Protein (CRP), arterial blood gases and levels of IL-6 or IL-8 [39]. Lipase levels are more sensitive and specific than those of amylase. There may be hyperamylasemia in renal failure, parotitis, ischemia and intestinal obstruction, macroamylasemia and by use of multiple medications. The lipase levels can increase spontaneously in bacterial peritonitis, intestinal ischemia and esophagitis [40].

\subsection{Imaging tests}

The guidelines of the ACG recommend that all patients with suspected AP should have an abdominal USG performed, which is useful for diagnosis of biliary AP, but limited in the presence of superimposed intestinal gas and choledocholithiasis, and it is not useful to evaluate prognosis [41]. CT is the standard diagnostic method for evaluation of the prediction and prognosis of AP severity, and is diagnostic test of choice for differential diagnosis in patients with severe abdominal pain that mimic AP or with atypical symptoms and mild elevations of serum pancreatic enzymes, and in case of conservative therapeutic failure or in the context of clinical deterioration. The optimal time to do it is 72 - 96 hours after the onset of symptoms [42]. MRI is useful in cases of hypersensitivity to contrast medium and shows an advantage over CT to evaluate the main pancreatic duct and the presence of collections [43]. ERCP is used in cases of choledocholithiasis and is similar to magnetic resonance (MR) cholangiopancreatography, non-invasive method that does not it requires gadolinium [13].

\section{Types and Phases}

There are two types: Interstitial edematous AP: Occurs in $80-90 \%$ of cases. It consists of acute inflammation of the pancreatic parenchyma and/or peripancreatic without necrotic tissue identifiable by CT. It resolves during the first week. Necrotizing AP: Inflammation associated with pancreatic necrosis and or peripancreatic detectable by CT. It is the most aggressive form [44-46].

There are 2 phases, which can be overcome and they are an early phase that remits in 1 week and can extend up to 2 weeks, characterized by the SIRS and/or organ failure. A late 
phase that lasts for weeks or months and is characterized by signs of systemic inflammation, local and systemic complications, and/or persistent organ failure [45,46].

\section{Degrees of Severity}

It is important to define and stratify AP to: a) Identify potentially serious patients who require aggressive treatment at admission, b) Identify patients that merit referral for specialized care and c) Stratify these patients in subgroups in the presence of persistent organ failure and complications including local or systemic.

The Atlanta Classification defines them as follows:

Mild AP: Absence of organ failure and local or systemic complications. Resolves during week 1 , usually do not require imaging examinations and mortality is very rare.

Moderately Severe AP: Presence of transient organ failure or local or systemic complications. It can resolve in the first 48 hours (transient organ failure or acute liquid collection) without intervention or requires prolonged specialized attention (Sterile PAN without organ failure), resolving in week 2 or 3 , with morbidity and mortality $<8 \%$.

Severe AP: Presence of persistent organ failure (single or multiple) and one or more local or systemic complications. It can happen in the early phase (mortality of 36-50\%) or late [45-47].

\section{Prediction of Severity and Forecast}

The identification of severity upon admission is detrimental to a) Determine if the patient enters intermediate care or intensive care, $b$ ) Decide on the initiation of effective and timely therapy, and c) Assess the risk of morbidity and mortality [48]. It is established at entry and at 48 hours combining the following parameters:

\subsection{Clinical parameters}

Age $\geq 60$ years, pre-existing disease (score $\geq 2$ in the Charlson comorbidity index), obesity (BMI > 30) and prolonged intake of alcohol, increase the risk of complications or death [36]. A new model using intra-abdominal pressure and BMI has been shown to more accurately predict the severity of AP [49].

\subsection{Laboratory Studies}

Hemoconcentration and azotemia, or alteration of inflammatory markers (CRP> $150 \mathrm{mg}$ / L and IL-6, IL-8, IL-10) measure the decrease in intravascular volume for losses in the third space $[40,50]$. If the BUN, creatinine and elevated hematocrit are not restored to normal range after an aggressive resuscitation with fluids, then these are predictors of severe AP. Amylase and lipase values are not predictors of severity [40,51]. Serum biomarkers such as the activation peptide of urinary trypsin and serum amyloid A have been studied as predictors of early severity [51]. Quantitative assessment of histone levels within 48 hours of onset of abdominal pain can also help predict persistent organ failure and mortality in patients with AP [52].

\subsection{SIRS}

It is defined with more than 2 of the following values: temperature $<36^{\circ} \mathrm{C}$ or $>38^{\circ} \mathrm{C}$, heart rate $>90 / \mathrm{min}$, respiratory rate $>20 / \mathrm{min}$ and white blood cells $<4000$ or $>12000 / \mathrm{mm} 3$. It predicts AP severity at admission and at 48 hours. For mortality, it has a sensitivity of 77 to $89 \%$ and specificity of 79 to $86 \%$ [53]. Persistent SIRS associated with multiple organ failure has $25 \%$ mortality compared to transient SIRS, which is $8 \%$ [54].

\subsection{Scoring systems}

a) Ranson criteria: With $80 \%$ sensitivity in the first 48 hours. Values 11 factors ( 5 on admission and 6 at 48 hours), a value $\geq 3$ points is considered severe AP [55,56]. 
b) APACHE II and APACHE-O: APACHE II has a sensitivity of $95 \%$ when used daily in patients in intensive care and assesses 12 criteria. A score $\geq 8$ is considered as a risk of death, and the risk is greater as the score increases. The APACHE-O is used when the obesity factor is added to the score [57-60].

c) Modified Glasgow Criteria (Imrie): Has sensitivity of $80 \%$ when used in the first 48 hours. A value $\geq 3$ predicts severe AP [61,62].

d) BALI score: Evaluate 4 variables: $\mathrm{BUN} \geq 25 \mathrm{mg} / \mathrm{dL}$, age $\geq 65$ years, $\mathrm{LDH} \geq 300 \mathrm{U} / \mathrm{L}$, IL-6 $\geq 300 \mathrm{pg} / \mathrm{mL}$, at admission and at 48 hours. Three positive variables are associated with mortality $\geq 25 \%$ and four positive variables with $\geq 50 \%$ [44].

e) PANC 3 Score: Very useful for its efficiency, ease and speed of results when evaluating 3 variables: Hematocrit $>44 \mathrm{~g} / \mathrm{dL}, \mathrm{BMI}>30 \mathrm{~kg} / \mathrm{m} 2$ and pleural effusion by $\mathrm{x}$-ray (this last variable is the most useful for predicting severe AP) [63].

Three recent scoring systems have been proposed, and each of them predicts severity in the first 24 hours [64].

f) BISAP: Evaluate 5 criteria: $\mathrm{BUN}>25 \mathrm{mg} / \mathrm{dL}$, age $>60$ years, mental deterioration, SIRS and pleural effusion. A value $>2$ elevates 10 times the risk of mortality.

g) HAPS: Identifies patients who do not require intensive care and that probably will not develop severe AP in 24 hours [63].

h) POP: Has greater sensitivity than APACHE II and Glasgow. Evaluate 6 variables: age, mean arterial pressure, arterial $\mathrm{pH}$, urea, calcium, $\mathrm{PaO} 2 / \mathrm{FIO} 2$. The score goes from $0-40$; a higher score equals higher mortality $[53,63,65]$.

There are other scoring systems such as:

i) Determinant-Based System: It is based on the identification of radiological changes of the collections, sterile PAN or infected and organ failure signs [66]. j) Severity index by CT: Sums the Balthazar degree, more the degree of necrosis $[56,58,62,67]$. Evaluates the presence of peripancreatic inflammation, phlegmon, and necrosis. A total $\geq 5$ is associated with prolonged hospital stay and increase in morbidity and mortality 15 times more than patients with a score $<5$. Exceeds Ranson's prediction of severity and APACHE II, has a sensitivity of $87 \%$ and specificity of $83 \%$.

k) Classification of Atlanta: It is based on a multifactorial scoring system and predictive factors of severity. In the first 24 hours includes clinical suspicion, an increase of BMI, pleural effusion and increased APACHE value II. After 24 hours it includes persistent organ failure and/or Glasgow Imrie $>3$ and higher severity score if CRP $>150 \mathrm{mg} / \mathrm{L}$ or increased biomarkers IL-1, IL-6, IL-8, IL- 10, procalcitonin and beta-receptor antagonist [45,46,58,63,68].

1) EPIC: Evaluates the presence of ascites, pleural effusion, and retroperitoneal edema. Predicts the occurrence of early organ failure with similar or greater precision to SIRS, Bisap and Balthazar, and duration of the hospital stay. It is not useful to differentiate the severity of organ failure, and the number of organs failed [69].

There is no reliable predictor or consensus on the preference of use of one or the other of the systems for persistent organ failure. Despite the evidence, there are no studies with a direct relationship between prognostic and mortality markers [69]. Based on expert opinions we can say that Ranson's criteria although simple, easy to remember and widely available tests to perform them in any laboratory, it is filled with uncertainties, uncomfortable and confusing as they vary according to the biliary etiology, and instead of evaluating 11 factors become 22 . All scoring systems have their utility but with the limitations that most are evaluated on admission and then until 48 hours, leaving a blind period that is critical in the management and detection of complications. The APACHE II Score has the advantage of being able to be calculated on admission and at any time according to the evolution and condition of the patient, is very effective for the accuracy of prediction of severity and prognosis, and together with the CT Severity Index are superior to the other numerous methods, since it also values extension and necrosis. Therefore we could conclude that the Ranson Criteria does have its utility when approaching the patient in the Emergency Room or on admission, but definitely, the APACHE II Score is the basis for constant clinical-laboratory combined monitoring $[58,59,61,63]$. 


\section{Treatment}

It is essential to make an accurate diagnosis, triage appropriately, provide high-quality supportive care, continuously monitor and treat complications, and prevent relapse $[1,70,71]$. Mild AP can be treated on an outpatient basis with oral analgesia; however, most require hospitalization [70]. The first 48-72 hours should focus on detecting monitoring worsening blood pressure, oxygen saturation and decreasing urine output. The presence of hypotension, tachycardia, hypoxemia, and oliguria $>48$ hours indicates persistent organ failure, and if not responding with adequate IV fluid therapy requires management in ICU and probably involving gastroenterology or general surgery [6]. The physical examination must be repeated every 4- 8 hours, monitoring for alteration of the mental state and/or abdominal stiffness indicating fluid in the third space or abdominal compartment syndrome [72,73]. In the first 612 hours complete metabolic panel, complete blood count, serum calcium, magnesium, glucose, and BUN, depending on the patient's condition should be performed. Hypocalcemia and hypomagnesemia should be corrected intravenously. Hyperglycemia must be managed with insulin. Hemoconcentration and elevated BUN levels indicate inadequate hydration or kidney injury, which requires increasing the supply of IV fluids Abdominal CT should be repeated if there is poor response to standard therapy to evaluate complications or worsening of the condition [68].

\subsection{Pain control}

Opioids are the analgesics of choice. They have the advantage of decrease the need for supplemental analgesia compared with other options, although there is no difference in risk of complications or serious adverse events. Pain medications used are buprenorphine, pethidine, pentazocine, fentanyl and morphine [74,75].

\subsection{Resuscitation with fluids}

The substantial losses in the third space and the depletion of intravascular volume (hypovolemia) leading to hypoperfusion of the splenic bed are negative predictors for AP (hemoconcentration and azotemia) [76]. Retrospective studies suggest that the aggressive administration of fluids during the first 24 hours reduces morbidity and mortality. Most of the current guidelines provide guidelines for the administration of early and vigorous IV fluids, which is more important during the first 12-24 hours and of little value after this time.

Studies from Mayo Clinic in the United States have demonstrated that with the abovementioned management, there is a decrease in the incidence of organ failure and the SIRS score, and length of hospital/ICU [76]. The AGA recommends administering balanced crystalloid solution at $200-500 \mathrm{~mL} /$ hour or $5-10 \mathrm{~mL} / \mathrm{Kg}$ weight/hour $(2500-4000 \mathrm{~mL}$ in the first 24 hours) to maintain an effective MAP (65 mmHg) and a urinary output of $0.5 \mathrm{~mL} / \mathrm{Kg}$ weight/hour, and so on to decrease BUN levels [5]. Ringer's Lactate is superior to normal saline solution and reduces more inflammatory markers (CRP) and the incidence of SIRS [77,78]. An experimental study in rats with PAN using solution of Ringer's ethyl pyruvate (replacing Lactate) showed to be a powerful antioxidant that decreased edema and pancreatic necrosis achieving reduction of terminal organ failure and improvement in survival [76].

Colloids should be considered in case of hematocrit $<25 \%$ and human albumin for hypoalbuminemia $<2 \mathrm{~g} / \mathrm{dL}$ [64]. The practical way to measure the adequacy of fluid therapy and of the state of hydration is by cardiopulmonary monitoring, clinically and hourly measurement of urine output, monitoring of hematocrit (of 35-44\%) and correction of BUN and creatinine, which has shown that it limits necrosis. A decrease in hematocrit level to $44-47 \%$ in the first 24 hours is considered a major risk to develop necrosis [64]. The main risk of fluid restitution is the overload of volume that increases the risk of abdominal compartment syndrome, sepsis, need for intubation and death $[41,73]$, so it must be adapted according to the degree of intravascular volume depletion and cardiopulmonary reserve, having special considerations in patients with renal and cardiopulmonary failure [64]. 


\subsection{Nutrition}

In the last decade, nutritional support has become one of the key points in the treatment of AP, mainly of severe AP. There is an indication of specialized nutrition, with the enteral nutrition (EN) being preferred over the total parenteral nutrition (TPN) administered from the early form. The use of polymeric diets is recommended, where the three basic protein nutrients are present, carbohydrates and fats, accompanied by clear liquids. TPN is more expensive, riskier and less effective than the EN in AP patients and is reserved as a second line. In mild AP without organ failure or necrosis, EN can be started from admission with a soft or solid diet low in fat, in the absence of intense pain, nausea, vomiting, and ileus, without waiting for normalization of the levels of pancreatic enzymes. It is safe and associated with a shorter hospital stay compared to a clear liquid diet progressing slowly to a solid diet [76,79]. In severe AP, intolerance to the oral route or when the clinical signs of AP worsen with EN, TPN is indicated; even so, it is recommended to maintain minimum perfusion of EN to preserve the trophic effect of the intestinal mucosa. Can start EN when sufficient oral intake is not reached in 3 to 5 days, by nasojejunal tube (Dobhoff) which is better than the nasogastric because it decreases the pancreatic secretion, prevents the risk of bacterial translocation of intestine to the pancreas and infections, decreases the need for surgical interventions and short hospital stay/ICU, independently of the APACHE II score, without changes in the occurrence of complications and mortality, compared with the TPN. A recent meta-analysis also showed reduction in the mortality and organ failure [76,79-81]. In AP, regardless of the severity, a normal diet can be started once the acute pain is resolving [82].

\subsection{Antibiotics}

AP is a sterile inflammation; only one-third of cases develop infected PAN with a significant risk of death $>50 \%$. The use of antibiotics should be reserved for suspicion or confirmation of local or extrapancreatic infections such as pneumonia, urinary tract infection, cholangitis, sepsis, phlebitis in a venipuncture site; or as recommended by the guidelines of AGA and ACG clinics, restricting them to patients with PAN with pancreatic necrosis $>30 \%$ or pancreatic necrosis or infected extrapancreatic, which should be suspected based on clinical deterioration or poor patient improvement after 7-10 days of hospital treatment [41]. They are also indicated in sepsis, so simultaneous pan cultures (blood, urine, other bodily and tracheal fluids), should be done to identify etiology. Antibiotic administration must be done according to sensitivity, and the duration depends on the clinical response and negativity of the cultures [83-85]. The use of prophylactic antibiotics is not indicated in mild AP, moderately severe, severe or sterile necrosis which increases the prevalence of fungal infections and development of multidrug-resistant microorganisms [83-86].

Various meta-analysis and reviews in Cochrane conclude that the carbapenems, especially imipenem/cilastatin, are the empirical monotherapy more effective for the treatment of pancreatic infection. Quinolones, metronidazole, and cephalosporins at high doses can also be effective since most isolated pathogens are E. coli, Bacteroides, Enterobacter, Klebsiella, S. faecalis, S. epidermidis, and S. aureus [87].

\subsection{Probiotics and prebiotics}

The Dutch AP Study Group found that the use of a probiotic cocktail increased death with high significance statistics, therefore, are contraindicated. The same happens with prebiotics $[88,89]$.

\subsection{Alternative medicine}

A Cochrane review evaluated 15 Chinese studies determining that the use in AP of licorice root, ginseng, peony and bark of Chinese cinnamon reduce mortality, surgical interventions, and organ failure, but the quality of the studies is very low [90,91]. 


\subsection{Other drugs}

The use of heparin (preferably those of low molecular weight) in moderately severe to severe AP is associated with better evolution by a lower incidence of necrosis, ability to improve pancreatic microcirculation and anti-inflammatory effect when reducing the stimulation of macrophages and monocytes [92]. The review by Rawla et al. has shown that heparin reduces amylase levels, endothelin-1, inflammatory cytokines and TNF- $\alpha$, and activation of NF-kB [12]. Simvastatin is a promising drug for the prophylaxis of new episodes of recurrent AP [12]. Various clinical trials demonstrate the benefit of the use of anti-TNF- $\alpha$ in selected patients since the TNF- $\alpha$ plays a central role in the pathogenesis of local and systemic complications of PA (4). Norepinephrine, as in patients with sepsis, is the first line vasopressor to maintain MAP $\geq 65$ $\mathrm{mmHg}$, even when hypovolemia has not yet been treated. Continuous Hemodiafiltration (CHDF) vs. Continuous Veno-Venous Hemofiltration (CVVH) is also used in some patients [93]. CHDF is widely used in Japan as a therapy for blood purification in patients with morbid conditions, and it is believed to prevent organ failure. Its role has been investigated using a membrane of Polymethyl methacrylate (PMMA) for the elimination of cytokines proinflammatory in severe AP. Pupelis et al., in a 10-year retrospective review of experience using $\mathrm{CVVH}$ in patients with $\mathrm{AP}$, concluded that balancing the replacement of fluids and the elimination of blood cytokines decreases the need of surgical interventions from $41 \%$ to $19 \%$ $[94,95]$. CVVH therapy can reduce the levels of inflammatory factors in severe AP patients effectively [96]. The Japanese guidelines suggest that organ failure is prevented but not mortality in severe AP. $\mathrm{Xu}$ et al. recently showed a decrease in the levels of TNF- $\alpha$ and the positive correlation between blood levels of TNF- $\alpha$ and the intra-abdominal pressure [97]. Use of pyruvate and galactose feedings have been shown to be protective against cell damage in AP when tested mouse models [98].

\subsection{Heparanase inhibitors}

A clinical-biochemical model of AP induction was studied in rats with analogues of interstitial pancreatic secretagogues, specifically cerulein, which increases expression and activity of heparanase (endoglycoside regulator of several physiological and pathological processes, as well as angiogenesis, metastatic cancer and inflammation), which is highly involved in its pathogenesis and the use of its inhibitors decrease edema and inflammation markedly. Currently, they are used in phase I / II of clinical trials in cancer patients, so they are expected to be also beneficial in AP [99].

\subsection{Endoscopic treatment}

ERCP with sphincterotomy decreases mortality and complications compared with nonsphincterotomized cases. Its use is limited to patients with over-aggregated cholangitis. Biliary AP should be treated urgently preferably in the first 24 hours when there is documented choledocholithiasis by images or findings highly suggestive of persistent calculation in the bile duct, for example, jaundice, progressive increase in liver function tests or persistent dilation of the bile duct. There is no benefit in the absence of these manifestations, mild biliary AP or as a diagnostic test before cholecystectomy. Endoscopic ultrasonography (EUS) is used as a platform for the minimally invasive treatment of pseudocyst with necrosis of the pancreatic wall [100102].

\section{Complications}

\subsection{Organic failure}

It is defined for 3 systems (respiratory: $\mathrm{PaO} 2 / \mathrm{FiO} 2=300$, cardiovascular: use of inotropic agents and renal: creatinine $=171 \mathrm{mmol} / \mathrm{L}$ or $2.0 \mathrm{mg} / \mathrm{dL}$ ) in a 24 -hour period and a score $\geq 2$ with Marshall's modified system that is preferable to the SOFA system since it is more 
simplified, universal and stratifies severity especially in critically ill patients with inotropic and ventilatory support; both can be used on admission and daily . Organ failure may be transient if it resolves in $<48$ hours, and persistent if it is $\geq 48$ hours, with mortality up to $30 \%$ [44,68].

\subsection{Systemic complications}

a) Exacerbation of pre-existing comorbidities: Coronary disease, chronic liver disease, COPD, acute renal failure and intravascular disseminated coagulation. b) Abdominal compartment syndrome: Defined as a concomitant organ failure with intra-abdominal pressure $>20 \mathrm{~mm} \mathrm{Hg}$ as a consequence of aggressive resuscitation with fluid, manifested with abdominal distension, oliguria or increase in assisted mechanical ventilation. Its management consists of a) Decrease the contribution of liquids, b) Measure intra-vesical pressure with a urinary catheter, c) Reduce the ventilatory tidal volume and d) Place nasogastric and rectal tubes. If these measures are not effective, then surgical decompression is preferred [73,103].

c) After AP endocrine dysfunction (prediabetes and DM2) appears in 20-30\% and the exocrine $(\mathrm{CP})$ in one third or half of the cases. $60 \%$ of Patients with DM2 are not associated with pancreatic exocrine dysfunction because it recovers over time [104].

d) Other complications: Thrombosis of the portal vein, ascites by thrombosis of the superior mesenteric vein and/or Splenic aneurysm may occur after several weeks of hospitalization. Gastric dysfunction, necrosis of the colon, acidosis, Respiratory distress syndrome, gastric varices, retroperitoneal hemorrhage and gastrointestinal, ileus, pleural effusion, aneurysms in the splenic, renal or gastroduodenal arteries and gastric hematoma have also been reported [71].

\subsection{Local complications}

They are suspected when persistent abdominal pain or recurrent increased pancreatic enzymes are seen with organ failure and/or signs of SIRS. By themselves, they do not define AP severity. They are described by CT based on location (pancreatic, peripancreatic or other), the content (liquid, solid or gas) and thickness of the wall (thin or thick) [105]. Currently, abdominal CT is a non-invasive diagnostic tool to detect collections $[56,106]$. There are four types of collections, which appear in this order: 1. Acute liquid collection: Before 4 weeks. Associated with interstitial edematous AP in the absence of necrosis [76]. It is homogeneous with dense liquid, not encapsulated and confined to the peripancreatic fascia. Most are sterile and have a spontaneous resolution, so their management is conservative. 2. Acute necrotic collection or PAN: In the first 4 weeks. It can be sterile or infected. In the initials phases the collection is a mixture of solid tissue and semi-solid, then it becomes more liquid, and it is encapsulated (PAN encapsulated sterile). Infected PAN is rare in the first 2 weeks, and it is suspected after this time before the onset of fever, leukocytosis, and pain. Abdominal growth is generally monomicrobial and more frequent by gram-negative bacilli. CT demonstrates gas in the presence of necrosis. If sterile, it is managed conservatively and only requires therapy in rare cases that obstructs a nearby viscus, for example, stomach, duodenum or bile duct $[86,107,108]$.

In current practice any invasive intervention is delayed for at least 4 weeks; when encapsulation demarcates the boundary between the necrotic tissue and healthy, forming a mature wall around it which allows drainage and debridement easier, reducing the risk of complications and death. If the patient has progressive sepsis or is in unstable conditions, the percutaneous drainage of the collection is enough to reduce sepsis and allow delay of 4 weeks to be continued. Almost $60 \%$ of patients with PAN can be treated non-invasively with a low risk of death [86].

In patients who develop severe AP and infected PAN or persistent liquid collections, the treatment consists of administering antibiotics, CT-guided percutaneous drainage if necessary and after a delay of several weeks performs minimally invasive debridement (necrosectomy) by percutaneous, endoscopic, laparoscopic or assisted retroperitoneal approach. This approach is superior to traditional open necrosectomy with respect to the risk of major complications or death, and approximately one-third of patients treated with this method do not require 
debridement. The drained liquid is Gram stained and cultured. Only a small percentage of patients with infected PAN can be treated only with antibiotics $[86,107]$.

3. Pseudocyst: Appears around 4 weeks. Is an oval or round collection, encapsulated by a fibrous wall and well-defined granulation extrapancreatic tissue without necrosis or with minimal necrosis with a homogeneous composition. They are usually asymptomatic; when there are symptoms, they are nonspecific. If it is asymptomatic, it is managed conservatively, since $>50 \%$ resolve spontaneously. If it is symptomatic, it becomes infected or increases in size on serial images, should be intervened mainly with endoscopic techniques [105,109,110]. 4 . Abscess: After 4 weeks in the context of PAN, it is heterogeneous and encapsulated. More than $80 \%$ of the AP deaths are attributed to septic complications of PAN with bacterial infection. It is suspected by the clinical evolution of the patient or the presence of gas. Its management is similar to infected PAN [111]. Other local complications are: Pancreatic fistula, pseudocyst or pancreatocutaneous fistula, pseudoaneurysm of the pancreatic artery, portal vein thrombosis, superior mesenteric (manifested by ascites of recent onset) and/or splenic aneurysm can occur many weeks after hospitalization and should be detected and treated adequate and timely [71,105]. Hemorrhage of a pancreatic bed or retroperitoneum should be managed according to the etiology. In the case of aneurysm rupture, embolization is indicated, and if it does not resolve, surgery should be performed. Intramural hematomas in the absence of bleeding or other complications are handled conservatively. Otherwise, embolization is a safe and minimally invasive option, reserving surgery for cases selected [112-114].

\section{Surgical treatment}

In patients with mild biliary AP, cholecystectomy should be performed early (first 48 hours after admission), shortening hospital stay when comparing it to when it is done after the resolution of pain and normalization of enzymes. Delay increases the risk of recurrent biliary AP but not complications $[115,116]$. Biliary PAN surgery is delayed until the inflammation, and liquid collections stabilize, decrease or disappear, around 6 weeks $[116,117]$.

\section{Recurrent AP}

It is characterized by episodes of AP in more than one occasion by normal morphofunctional adjustment of the gland. However, $\mathrm{CP}$ can be found in both the first episode of AP as in the follow-up. It is idiopathic in $30 \%$ of the cases, but in the majority the cause is identified: choledocholithiasis or sludge and bile crystals in the common bile duct, dysfunction of the sphincter of Oddi, anatomical ductal variants that interfere with the exit of pancreatic juice, obstruction of the duct pancreatic main or pancreaticobiliary junction, genetic mutations and prolonged consumption of alcohol. In $80 \%$ of cases, cholecystectomy and eventually ERCP with Sphincterotomy are used for management. Ursodeoxycholic acid is also effective for the treatment of biliary sludge $[1,118,119]$.

\section{Prevention of Relapse}

The rate of relapse of AP is $20 \%$ at 30 days. It is important to emphasize that recurrent abdominal pain is an essential factor for relapse. Risk factors include in the first episode: severity, the degree of necrosis and cause, as well as the use of antibiotics. The factors that reduce it are tolerable solid diet and absence of gastrointestinal symptoms such as nausea, vomiting, diarrhea and abdominal pain. The most common modifiable factors are alcohol consumption and tobacco abuse. It has been seen that patients who continue to consume alcohol have a higher risk. Control of hyperlipidemias can prevent it. Finally, with biliary AP, a cholecystectomy should be performed before hospital discharge, reducing the complication rate by $75 \%$. If it is deferred, it should be done in a short ambulatory period and not for more than a few weeks, as the chances of recurrences increase to $>30 \%$. In patients with severe PAN or AP, cholecystectomy is postponed until inflammation decreases and improve visibility at the 
moment of surgery. Patients who are not candidates for surgery, biliary endoscopy can help reduce but not eliminate the recurrent risk of acute cholecystitis or biliary colic $[34,120]$.

\section{Conclusions:}

The main etiologic causes of AP are alcohol consumption, gallstones, hypertriglyceridemia, and biliary stones. The clinical signs and symptoms, and diagnostic criteria of AP are well established in the literature and multiple studies. Multiple scoring systems have been used to predict the severity, prognosis, and mortality associated with AP. We could conclude that the Ranson Criteria does have its utility when approaching the patient in the Emergency Room or on admission, but definitely, the APACHE II Score is the basis for constant clinical-laboratory combined monitoring. Early and vigorous IV fluids mainly with ringers' lactate is important during the first 12-24 hours of management of AP. In patients with mild biliary AP, cholecystectomy should be performed early (first 48 hours after admission), shortening hospital stay. Larger trials are needed on the role of heparinase inhibitors in the management of acute pancreatitis.

Supplementary Materials: None.

Author Contributions: All the authors have made substantive contributions to the article and assume full responsibility for its content

Funding: This research received no external funding.

Acknowledgments: None

Conflicts of Interest: The authors declare no conflict of interest."

\section{References:}

1. Testoni, P.A. Acute recurrent pancreatitis: Etiopathogenesis, diagnosis and treatment. World Journal of Gastroenterology 2014, 20, 16891, doi:10.3748/wjg.v20.i45.16891.

2. Portelli, M.; Jones, C.D. Severe acute pancreatitis: pathogenesis, diagnosis and surgical management. Hepatobiliary \& Pancreatic Diseases International 2017, 16, 155-159, doi:10.1016/s1499-3872(16)60163-7.

3. Gullo, L.; Migliori, M.; Oláh, A.; Farkas, G.; Levy, P.; Arvanitakis, C.; Lankisch, P.; Beger, H. Acute Pancreatitis in Five European Countries: Etiology and Mortality. Pancreas 2002, 24, 223-227, doi:10.1097/00006676-200204000-00003.

4. Xiao, A.Y.; Tan, M.L.Y.; Wu, L.M.; Asrani, V.M.; Windsor, J.A.; Yadav, D.; Petrov, M.S. Global incidence and mortality of pancreatic diseases: a systematic review, meta-analysis, and meta-regression of population-based cohort studies. The Lancet Gastroenterology \& Hepatology 2016, 1, 45-55, doi:10.1016/s24681253(16)30004-8.

5. Sultan, S.; Falck-Ytter, Y.; Inadomi, J.M. The AGA Institute Process for Developing Clinical Practice Guidelines Part One: Grading the Evidence. Clinical Gastroenterology and Hepatology 2013, 11, 329-332, doi:10.1016/j.cgh.2013.02.001.

6. Greenberg, J.A.; Hsu, J.; Bawazeer, M.; Marshall, J.; Friedrich, J.O.; Nathens, A.; Coburn, N.; May, G.R.; Pearsall, E.; McLeod, R.S. Clinical practice guideline: management of acute pancreatitis. Canadian Journal of Surgery 2016, 59, 128-140, doi:10.1503/cjs.015015.

7. IAP/APA evidence-based guidelines for the management of acute pancreatitis. Pancreatology 2013, 13, e1e15, doi:10.1016/j.pan.2013.07.063.

8. Yadav, D.; Lowenfels, A.B. Trends in the Epidemiology of the First Attack of Acute Pancreatitis. Pancreas 2006, 33, 323-330, doi:10.1097/01.mpa.0000236733.31617.52.

9. Tryliskyy, Y.; Bryce, G.J. Post-ERCP pancreatitis: Pathophysiology, early identification and risk stratification. Advances in clinical and experimental medicine : official organ Wroclaw Medical University 2018, 27, 149-154, doi:10.17219/acem/66773.

10. Forsmark, C.E.; Swaroop Vege, S.; Wilcox, C.M. Acute Pancreatitis. New England Journal of Medicine 2016, 375, 1972-1981, doi:10.1056/nejmra1505202.

11. Habtezion, A. Inflammation in acute and chronic pancreatitis. Current Opinion in Gastroenterology 2015, 31, 395-399, doi:10.1097/mog.0000000000000195. 
12. Rawla, P.; Sunkara, T.; Thandra, K.C.; Gaduputi, V. Hypertriglyceridemia-induced pancreatitis: updated review of current treatment and preventive strategies. Clinical journal of gastroenterology 2018, 11, 441-448, doi:10.1007/s12328-018-0881-1.

13. Mandalia, A.; Wamsteker, E.J.; DiMagno, M.J. Recent advances in understanding and managing acute pancreatitis. F1000Research 2018, 7, doi:10.12688/f1000research.14244.2.

14. Pang, Y.; Kartsonaki, C.; Turnbull, I.; Guo, Y.; Yang, L.; Bian, Z.; Chen, Y.; Millwood, I.Y.; Bragg, F.; Gong, W., et al. Metabolic and lifestyle risk factors for acute pancreatitis in Chinese adults: A prospective cohort study of 0.5 million people. PLoS medicine 2018, 15, e1002618, doi:10.1371/journal.pmed.1002618.

15. Greer, J.B.; Thrower, E.; Yadav, D. Epidemiologic and Mechanistic Associations Between Smoking and Pancreatitis. Current treatment options in gastroenterology 2015, 13, 332-346, doi:10.1007/s11938-015-0056-9.

16. Alsamarrai, A.; Das, S.L.M.; Windsor, J.A.; Petrov, M.S. Factors That Affect Risk for Pancreatic Disease in the General Population: A Systematic Review and Meta-analysis of Prospective Cohort Studies. Clinical Gastroenterology and Hepatology 2014, 12, 1635-1644.e1635, doi:10.1016/j.cgh.2014.01.038.

17. Ye, X.; Lu, G.; Huai, J.; Ding, J. Impact of smoking on the risk of pancreatitis: a systematic review and metaanalysis. PLoS One 2015, 10, e0124075, doi:10.1371/journal.pone.0124075.

18. Tenner, S. Drug induced acute pancreatitis: does it exist? World journal of gastroenterology 2014, 20, 1652916534, doi:10.3748/wjg.v20.i44.16529.

19. Rawla, P.; Raj, J.P. Doxycycline-Induced Acute Pancreatitis: A Rare Adverse Event. Gastroenterology research 2017, 10, 244-246, doi:10.14740/gr838w.

20. Hasan, A.; Moscoso, D.I.; Kastrinos, F. The Role of Genetics in Pancreatitis. Gastrointestinal endoscopy clinics of North America 2018, 28, 587-603, doi:10.1016/j.giec.2018.06.001.

21. Martinez-Barona, S.; Ribes-Koninckx, C. Genetics in idiopathic pancreatitis and acute recurrent pancreatitis. Revista espanola de enfermedades digestivas : organo oficial de la Sociedad Espanola de Patologia Digestiva 2017, 109, 478-479, doi:10.17235/reed.2017.4632/2016.

22. Sahin-Toth, M. Genetic risk in chronic pancreatitis: the misfolding-dependent pathway. Curr Opin Gastroenterol 2017, 33, 390-395, doi:10.1097/mog.0000000000000380.

23. Majumder, S.; Takahashi, N.; Chari, S.T. Autoimmune Pancreatitis. Digestive diseases and sciences 2017, 62, 1762-1769, doi:10.1007/s10620-017-4541-y.

24. Berger, Z.; Mancilla, C. Is autoimmune pancreatitis a subclass of chronic pancreatitis? Pancreatology 2017, 17, 55, doi:10.1016/j.pan.2016.12.008.

25. Wayne, M.; Delman, K.A.; Kurt, T.; Grossi, R.; Sabatini, M.; Cooperman, A. Autoimmune Pancreatitis: Unveiling a Hidden Entity. Archives of Surgery 2005, 140, 1104-1107, doi:10.1001/archsurg.140.11.1104.

26. Sureka, B.; Rastogi, A. Autoimmune Pancreatitis. Polish journal of radiology 2017, 82, 233-239, doi:10.12659/pjr.900899.

27. Hart, P.A.; Krishna, S.G.; Okazaki, K. Diagnosis and Management of Autoimmune Pancreatitis. Current treatment options in gastroenterology 2017, 15, 538-547, doi:10.1007/s11938-017-0147-x.

28. Hasanovic, J.; Agic, M.; Rifatbegovic, Z.; Mehmedovic, Z.; Jakubovic-Cickusic, A. Pancreatic injury in blunt abdominal trauma. Medical archives (Sarajevo, Bosnia and Herzegovina) 2015, 69, 130-132, doi:10.5455/medarh.2015.69.130-132.

29. Rawla, P.; Bandaru, S.S.; Vellipuram, A.R. Review of Infectious Etiology of Acute Pancreatitis. Gastroenterology Res 2017, 10, 153-158, doi:10.14740/gr858w.

30. Mikolasevic, I.; Milic, S.; Orlic, L.; Poropat, G.; Jakopcic, I.; Franjic, N.; Klanac, A.; Kristo, N.; Stimac, D. Metabolic syndrome and acute pancreatitis. European Journal of Internal Medicine 2016, 32, 79-83, doi:10.1016/j.ejim.2016.04.004.

31. Yoon, S.B.; Choi, M.H.; Lee, I.S.; Lim, C.-H.; Kim, J.S.; Cho, Y.K.; Park, J.M.; Lee, B.-I.; Cho, Y.-S.; Choi, M.G. Impact of body fat and muscle distribution on severity of acute pancreatitis. Pancreatology 2017, 17, 188193, doi:10.1016/j.pan.2017.02.002.

32. Binker, M.G. Acute pancreatitis: The stress factor. World Journal of Gastroenterology 2014, 20, 5801, doi:10.3748/wjg.v20.i19.5801.

33. Roberts, S.E.; Morrison-Rees, S.; John, A.; Williams, J.G.; Brown, T.H.; Samuel, D.G. The incidence and aetiology of acute pancreatitis across Europe. Pancreatology 2017, 17, 155-165, doi:10.1016/j.pan.2017.01.005.

34. Machicado, J.D.; Yadav, D. Epidemiology of Recurrent Acute and Chronic Pancreatitis: Similarities and Differences. Digestive diseases and sciences 2017, 62, 1683-1691, doi:10.1007/s10620-017-4510-5.

35. Dick, J.F.; Gardner, T.B.; Merrens, E.J. Acute pancreatitis: New developments and strategies for the hospitalist. Journal of Hospital Medicine 2016, 11, 724-729, doi:10.1002/jhm.2615. 
36. Phillip, V.; Steiner, J.M.; Algül, H. Early phase of acute pancreatitis: Assessment and management. World journal of gastrointestinal pathophysiology 2014, 5, 158-168, doi:10.4291/wjgp.v5.i3.158.

37. Valette, X.; du Cheyron, D. Cullen's and Grey Turner's Signs in Acute Pancreatitis. New England Journal of Medicine 2015, 373, e28, doi:10.1056/NEJMicm1504339.

38. Basnayake, C.; Ratnam, D. Blood tests for acute pancreatitis. Australian prescriber 2015, 38, 128-130, doi:10.18773/austprescr.2015.043.

39. Kaw, M.; Singh, S. Serum lipase, C-reactive protein, and interleukin-6 levels in ERCP-induced pancreatitis. Gastrointestinal endoscopy 2001, 54, 435-440.

40. Ismail, O.Z.; Bhayana, V. Lipase or amylase for the diagnosis of acute pancreatitis? Clinical biochemistry 2017, 50, 1275-1280, doi:10.1016/j.clinbiochem.2017.07.003.

41. Tenner, S.; Baillie, J.; DeWitt, J.; Vege, S.S. American College of Gastroenterology guideline: management of acute pancreatitis. The American journal of gastroenterology 2013, 108, 1400.

42. Arvanitakis, M.; Delhaye, M.; De Maertelaere, V.; Bali, M.; Winant, C.; Coppens, E.; Jeanmart, J.; Zalcman, M.; Van Gansbeke, D.; Devière, J., et al. Computed tomography and magnetic resonance imaging in the assessment of acute pancreatitis. Gastroenterology 2004, 126, 715-723, doi:10.1053/j.gastro.2003.12.006.

43. Štimac, D.; Miletić, D.; Radić, M.; Krznarić, I.; Mazur-Grbac, M.; Perković, D.; Milić, S.; Golubović, V. The Role of Nonenhanced Magnetic Resonance Imaging in the Early Assessment of Acute Pancreatitis. The American Journal of Gastroenterology 2007, 102, 997-1004, doi:10.1111/j.1572-0241.2007.01164.x.

44. Quinlan, J.D. Acute pancreatitis. American family physician 2014, 90, 632-639.

45. Banks, P.A.; Bollen, T.L.; Dervenis, C.; Gooszen, H.G.; Johnson, C.D.; Sarr, M.G.; Tsiotos, G.G.; Vege, S.S. Classification of acute pancreatitis-2012: revision of the Atlanta classification and definitions by international consensus. Gut 2012, 62, 102-111, doi:10.1136/gutjnl-2012-302779.

46. Sarr, M.G. 2012 revision of the Atlanta Classification of acute pancreatitis. Polish Archives of Internal Medicine 2013, 123, 118-124, doi:10.20452/pamw.1627.

47. Zaheer, A.; Singh, V.K.; Qureshi, R.O.; Fishman, E.K. The revised Atlanta classification for acute pancreatitis: updates in imaging terminology and guidelines. Abdominal Imaging 2012, 38, 125-136, doi:10.1007/s00261-012-9908-0.

48. Coffey, M.J.; Nightingale, S.; Ooi, C.Y. Tu1494 Early Prediction of Severity in Acute Paediatric Pancreatitis. Gastroenterology 2012, 142, S-848, doi:10.1016/s0016-5085(12)63288-0.

49. Fei, Y.; Gao, K.; Tu, J.; Wang, W.; Zong, G.-q.; Li, W.-q. Predicting and evaluation the severity in acute pancreatitis using a new modeling built on body mass index and intra-abdominal pressure. The American Journal of Surgery 2018, 216, 304-309, doi:10.1016/j.amjsurg.2017.04.017.

50. Lippi, G.; Valentino, M.; Cervellin, G. Laboratory diagnosis of acute pancreatitis: in search of the Holy Grail. Critical Reviews in Clinical Laboratory Sciences 2012, 49, 18-31, doi:10.3109/10408363.2012.658354.

51. Winslet, M.; Hall, C.; London, N.J.; Neoptolemos, J.P. Relation of diagnostic serum amylase levels to aetiology and severity of acute pancreatitis. Gut 1992, 33, 982-986, doi:10.1136/gut.33.7.982.

52. Liu, T.; Huang, W.; Szatmary, P.; Abrams, S.T.; Alhamdi, Y.; Lin, Z.; Greenhalf, W.; Wang, G.; Sutton, R.; Toh, C.H. Accuracy of circulating histones in predicting persistent organ failure and mortality in patients with acute pancreatitis. The British journal of surgery 2017, 104, 1215-1225, doi:10.1002/bjs.10538.

53. Buxbaum, J.; Quezada, M.; Chong, B.; Gupta, N.; Yu, C.Y.; Lane, C.; Da, B.; Leung, K.; Shulman, I.; Pandol, S. The Pancreatitis Activity Scoring System predicts clinical outcomes in acute pancreatitis: findings from a prospective cohort study. The American journal of gastroenterology 2018, 113, 755.

54. Wilson, J.; Zarabi, S. BET 1: SIRS criteria as a way of predicting mortality in acute pancreatitis. Emergency Medicine Journal 2017, 34, 621-622, doi:10.1136/emermed-2017-207038.1.

55. Kiriyama, S.; Gabata, T.; Takada, T.; Hirata, K.; Yoshida, M.; Mayumi, T.; Hirota, M.; Kadoya, M.; Yamanouchi, E.; Hattori, T., et al. New diagnostic criteria of acute pancreatitis. Journal of Hepato-BiliaryPancreatic Sciences 2009, 17, 24-36, doi:10.1007/s00534-009-0214-3.

56. Aphinives, P.; Karunasumetta, C.; Bhudhisawasdi, V.; Saesaew, O.T. Acute pancreatitis: assessment severity with Ranson score and CT evaluation. Journal of the Medical Association of Thailand = Chotmaihet thangphaet 2011, 94, 437-440.

57. Rawla, P.; Thandra, K.C.; Sunkara, T. Pancreatic cancer and obesity: epidemiology, mechanism, and preventive strategies. Clinical journal of gastroenterology 2019, 10.1007/s12328-019-00953-3, doi:10.1007/s12328-019-00953-3. 
58. Harshit Kumar, A.; Singh Griwan, M. A comparison of APACHE II, BISAP, Ranson's score and modified CTSI in predicting the severity of acute pancreatitis based on the 2012 revised Atlanta Classification. Gastroenterology report 2018, 6, 127-131, doi:10.1093/gastro/gox029.

59. Yang, L.; Liu, J.; Xing, Y.; Du, L.; Chen, J.; Liu, X.; Hao, J. Comparison of BISAP, Ranson, MCTSI, and APACHE II in Predicting Severity and Prognoses of Hyperlipidemic Acute Pancreatitis in Chinese Patients. Gastroenterology research and practice 2016, 2016, 1834256-1834256, doi:10.1155/2016/1834256.

60. Chatzicostas, C.; Roussomoustakaki, M.; Vlachonikolis, I.G.; Notas, G.; Mouzas, I.; Samonakis, D.; Kouroumalis, E.A. Comparison of Ranson, APACHE II and APACHE III scoring systems in acute pancreatitis. Pancreas 2002, 25, 331-335.

61. Khanna, A.K.; Meher, S.; Prakash, S.; Tiwary, S.K.; Singh, U.; Srivastava, A.; Dixit, V.K. Comparison of Ranson, Glasgow, MOSS, SIRS, BISAP, APACHE-II, CTSI Scores, IL-6, CRP, and Procalcitonin in Predicting Severity, Organ Failure, Pancreatic Necrosis, and Mortality in Acute Pancreatitis. HPB surgery : a world journal of hepatic, pancreatic and biliary surgery 2013, 2013, 367581-367581, doi:10.1155/2013/367581.

62. Tan, Y.H.A.; Rafi, S.; Tyebally Fang, M.; Hwang, S.; Lim, E.W.; Ngu, J.; Tan, S.M. Validation of the modified Ranson versus Glasgow score for pancreatitis in a Singaporean population. ANZ journal of surgery 2017, 87, 700-703, doi:10.1111/ans.13139.

63. Gray, R.; Cagliani, J.; Amodu, L.I.; Nauka, P.; Villacres, B.; Santos, T.; Castenada, A.; Fishbein, J.; Ahmed, N.; Coppa, G., et al. Maximizing the Use of Scoring Systems in the Prediction of Outcomes in Acute Pancreatitis. Digestion 2019, 99, 166-171, doi:10.1159/000490887.

64. Aggarwal, A. Fluid resuscitation in acute pancreatitis. World Journal of Gastroenterology 2014, 20, 18092, doi:10.3748/wjg.v20.i48.18092.

65. Harrison, D.A.; D'Amico, G.; Singer, M. The Pancreatitis Outcome Prediction (POP) Score: A new prognostic index for patients with severe acute pancreatitis*. Critical Care Medicine 2007, 35, 1703-1708, doi:10.1097/01.ccm.0000269031.13283.c8.

66. Dellinger, E.P.; Forsmark, C.E.; Layer, P.; Lévy, P.; Maraví-Poma, E.; Petrov, M.S.; Shimosegawa, T.; Siriwardena, A.K.; Uomo, G.; Whitcomb, D.C., et al. Determinant-Based Classification of Acute Pancreatitis Severity. Annals of Surgery 2012, 256, 875-880, doi:10.1097/sla.0b013e318256f778.

67. Bollen, T.L.; Singh, V.K.; Maurer, R.; Repas, K.; van Es, H.W.; Banks, P.A.; Mortele, K.J. Comparative Evaluation of the Modified CT Severity Index and CT Severity Index in Assessing Severity of Acute Pancreatitis. American Journal of Roentgenology 2011, 197, 386-392, doi:10.2214/ajr.09.4025.

68. Chen, C.; Huang, Z.; Li, H.; Song, B.; Yuan, F. Evaluation of extrapancreatic inflammation on abdominal computed tomography as an early predictor of organ failure in acute pancreatitis as defined by the revised Atlanta classification. Medicine 2017, 96, e6517, doi:10.1097/md.0000000000006517.

69. Li, Y.; Zhao, Y.; Feng, L.; Guo, R. Comparison of the prognostic values of inflammation markers in patients with acute pancreatitis: a retrospective cohort study. BMJ Open 2017, 7, e013206, doi:10.1136/bmjopen-2016013206.

70. Vege, S.S.; DiMagno, M.J.; Forsmark, C.E.; Martel, M.; Barkun, A.N. Initial Medical Treatment of Acute Pancreatitis: American Gastroenterological Association Institute Technical Review. Gastroenterology 2018, 154, 1103-1139, doi:10.1053/j.gastro.2018.01.031.

71. Zerem, E. Treatment of severe acute pancreatitis and its complications. World Journal of Gastroenterology 2014, 20, 13879, doi:10.3748/wjg.v20.i38.13879.

72. Leppaniemi, A.; Johansson, K.; De Waele, J.J. Abdominal compartment syndrome and acute pancreatitis. Acta clinica Belgica 2007, 62 Suppl 1, 131-135.

73. Jaipuria, J.; Bhandari, V.; Chawla, A.S.; Singh, M. Intra-abdominal pressure: Time ripe to revise management guidelines of acute pancreatitis? World J Gastrointest Pathophysiol 2016, 7, 186-198, doi:10.4291/wjgp.v7.i1.186.

74. Goulden, M.R. The pain of chronic pancreatitis: a persistent clinical challenge. British journal of pain 2013, 7, 8-22, doi:10.1177/2049463713479230.

75. Gachago, C.; Draganov, P.V. Pain management in chronic pancreatitis. World J Gastroenterol 2008, 14, $3137-$ 3148.

76. John, B.; Srinivasan, G.; Venkatakrishnan, L.; Sambandam, S.; Singh, G.; Kaur, M.; Janarthan, K. Current concepts in the management of acute pancreatitis. Journal of Family Medicine and Primary Care 2016, 5, 752, doi:10.4103/2249-4863.201144.

77. Iqbal, U.; Anwar, H.; Scribani, M. Ringer's lactate versus normal saline in acute pancreatitis: A systematic review and meta-analysis. Journal of digestive diseases 2018, 19, 335-341, doi:10.1111/1751-2980.12606. 
78. de-Madaria, E.; Herrera-Marante, I.; Gonzalez-Camacho, V.; Bonjoch, L.; Quesada-Vazquez, N.; AlmentaSaavedra, I.; Miralles-Macia, C.; Acevedo-Piedra, N.G.; Roger-Ibanez, M.; Sanchez-Marin, C., et al. Fluid resuscitation with lactated Ringer's solution vs normal saline in acute pancreatitis: A triple-blind, randomized, controlled trial. United European Gastroenterol J 2018, 6, 63-72, doi:10.1177/2050640617707864.

79. Horibe, M.; Nishizawa, T.; Suzuki, H.; Minami, K.; Yahagi, N.; Iwasaki, E.; Kanai, T. Timing of oral refeeding in acute pancreatitis: A systematic review and meta-analysis. United European Gastroenterology Journal 2016, 4, 725-732, doi:10.1177/2050640615612368.

80. Hegazi, R.A.; DeWitt, T. Enteral nutrition and immune modulation of acute pancreatitis. World Journal of Gastroenterology 2014, 20, 16101, doi:10.3748/wjg.v20.i43.16101.

81. Oláh, A.; Jr, L.R. Enteral nutrition in acute pancreatitis: A review of the current evidence. World Journal of Gastroenterology 2014, 20, 16123, doi:10.3748/wjg.v20.i43.16123.

82. van Dijk, S.M.; Hallensleben, N.D.L.; van Santvoort, H.C.; Fockens, P.; van Goor, H.; Bruno, M.J.; Besselink, M.G. Acute pancreatitis: recent advances through randomised trials. Gut 2017, 66, 2024-2032, doi:10.1136/gutjnl-2016-313595.

83. Ignatavicius, P.; Vitkauskiene, A.; Pundzius, J.; Dambrauskas, Z.; Barauskas, G. Effects of prophylactic antibiotics in acute pancreatitis. HPB : the official journal of the International Hepato Pancreato Biliary Association 2012, 14, 396-402, doi:10.1111/j.1477-2574.2012.00464.x.

84. Jiang, K.; Huang, W.; Yang, X.N.; Xia, Q. Present and future of prophylactic antibiotics for severe acute pancreatitis. World J Gastroenterol 2012, 18, 279-284, doi:10.3748/wjg.v18.i3.279.

85. Mourad, M.M.; Evans, R.; Kalidindi, V.; Navaratnam, R.; Dvorkin, L.; Bramhall, S.R. Prophylactic antibiotics in acute pancreatitis: endless debate. Annals of the Royal College of Surgeons of England 2017, 99, 107-112, doi:10.1308/rcsann.2016.0355.

86. Boumitri, C.; Brown, E.; Kahaleh, M. Necrotizing Pancreatitis: Current Management and Therapies. Clinical endoscopy 2017, 50, 357-365, doi:10.5946/ce.2016.152.

87. Dellinger, E.P.; Tellado, J.M.; Soto, N.E.; Ashley, S.W.; Barie, P.S.; Dugernier, T.; Imrie, C.W.; Johnson, C.D.; Knaebel, H.P.; Laterre, P.F., et al. Early antibiotic treatment for severe acute necrotizing pancreatitis: a randomized, double-blind, placebo-controlled study. Ann Surg 2007, 245, 674-683, doi:10.1097/01.sla.0000250414.09255.84.

88. Zhang, M.M.; Cheng, J.Q.; Lu, Y.R.; Yi, Z.H.; Yang, P.; Wu, X.T. Use of pre-, pro- and synbiotics in patients with acute pancreatitis: a meta-analysis. World J Gastroenterol 2010, 16, 3970-3978.

89. Gou, S.; Yang, Z.; Liu, T.; Wu, H.; Wang, C. Use of probiotics in the treatment of severe acute pancreatitis: a systematic review and meta-analysis of randomized controlled trials. Critical care 2014, 18, R57, doi:10.1186/cc13809.

90. Yue, Q.; Gao, G.; Zou, G.; Yu, H.; Zheng, X. Natural Products as Adjunctive Treatment for Pancreatic Cancer: Recent Trends and Advancements. BioMed research international 2017, 2017, 8412508, doi:10.1155/2017/8412508.

91. Stigliano, S.; Archibugi, L.; Zerboni, G.; Delle Fave, G.; Capurso, G. The Use of Complementary and Alternative Medicine is Frequent in Patients With Pancreatic Disorders. Journal of clinical gastroenterology 2016, 50 Suppl 2, Proceedings from the 8th Probiotics, Prebiotics \& New Foods for Microbiota and Human Health meeting held in Rome, Italy on September 13-15, 2015, S161-S163, doi:10.1097/MCG.0000000000000685.

92. Ceranowicz, P.; Dembinski, A.; Warzecha, Z.; Dembinski, M.; Cieszkowski, J.; Rembisz, K.; Konturek, S.J.; Kusnierz-Cabala, B.; Tomaszewska, R.; Pawlik, W.W. Protective and therapeutic effect of heparin in acute pancreatitis. Journal of physiology and pharmacology : an official journal of the Polish Physiological Society 2008, 59 Suppl 4, 103-125.

93. Cheng, Y.; Gong, J.; Ding, X.; Liu, Z.; Gong, J.; Zeng, Z. Continuous veno-venous hemofiltration for severe acute pancreatitis. Cochrane Database of Systematic Reviews 2018, 10.1002/14651858.CD012959, doi:10.1002/14651858.CD012959.

94. Pupelis, G.; Plaudis, H.; Grigane, A.; Zeiza, K.; Purmalis, G. Continuous veno-venous haemofiltration in the treatment of severe acute pancreatitis: 6-year experience. HPB : the official journal of the International Hepato Pancreato Biliary Association 2007, 9, 295-301, doi:10.1080/13651820701329225.

95. Pupelis, G.; Plaudis, H.; Zeiza, K.; Drozdova, N.; Mukans, M.; Kazaka, I. Early continuous veno-venous haemofiltration in the management of severe acute pancreatitis complicated with intra-abdominal hypertension: retrospective review of 10 years' experience. Annals of intensive care 2012, 2 Suppl 1, S21, doi:10.1186/2110-5820-2-S1-S21. 
96. Chen, X.; Sun, M.; Mao, X.; Liu, X.; Sun, W. Effectiveness of continuous veno-venous hemofiltration in the treatment of severe acute pancreatitis. Experimental and Therapeutic Medicine 2019, 17, 2720-2724.

97. Xu, J.; Tian, X.; Zhang, C.; Wang, M.; Li, Y. Management of abdominal compartment syndrome in severe acute pancreatitis patients with early continuous veno-venous hemofiltration. Hepato-gastroenterology 2013, 60, 1749-1752, doi:10.5754/hge13351.

98. Peng, S.; Gerasimenko, J.V.; Tsugorka, T.M.; Gryshchenko, O.; Samarasinghe, S.; Petersen, O.H.; Gerasimenko, O.V. Galactose protects against cell damage in mouse models of acute pancreatitis. The Journal of clinical investigation 2018, 128, 3769-3778, doi:10.1172/JCI94714.

99. Khamaysi, I.; Singh, P.; Nasser, S.; Awad, H.; Chowers, Y.; Sabo, E.; Hammond, E.; Gralnek, I.; Minkov, I.; Noseda, A., et al. The Role of Heparanase in the Pathogenesis of Acute Pancreatitis: A Potential Therapeutic Target. Scientific Reports 2017, 7, doi:10.1038/s41598-017-00715-6.

100. Fiocca, F.; Santagati, A.; Ceci, V.; Donatelli, G.; Pasqualini, M.J.; Moretti, M.G.; Speranza, V.; Di Giuli, M.; Minervini, S.; Sportelli, G., et al. ERCP and acute pancreatitis. European review for medical and pharmacological sciences 2002, 6, 13-17.

101. Canlas, K.R.; Branch, M.S. Role of endoscopic retrograde cholangiopancreatography in acute pancreatitis. World J Gastroenterol 2007, 13, 6314-6320.

102. Lee, H.S.; Chung, M.J.; Park, J.Y.; Bang, S.; Park, S.W.; Song, S.Y.; Chung, J.B. Urgent endoscopic retrograde cholangiopancreatography is not superior to early ERCP in acute biliary pancreatitis with biliary obstruction without cholangitis. PLoS One 2018, 13, e0190835, doi:10.1371/journal.pone.0190835.

103. De Waele, J.J.; Leppäniemi, A.K. Intra-Abdominal Hypertension in Acute Pancreatitis. World Journal of Surgery 2009, 33, 1128-1133, doi:10.1007/s00268-009-9994-5.

104. Das, S.L.M. Relationship between the exocrine and endocrine pancreas after acute pancreatitis. World Journal of Gastroenterology 2014, 20, 17196, doi:10.3748/wjg.v20.i45.17196.

105. Upchurch, E. Local complications of acute pancreatitis. British journal of hospital medicine 2014, 75, 698-702, doi:10.12968/hmed.2014.75.12.698.

106. Balthazar, E.J. Acute Pancreatitis: Assessment of Severity with Clinical and CT Evaluation. Radiology 2002, 223, 603-613, doi:10.1148/radiol.2233010680.

107. Buchler, M.W.; Gloor, B.; Muller, C.A.; Friess, H.; Seiler, C.A.; Uhl, W. Acute necrotizing pancreatitis: treatment strategy according to the status of infection. Ann Surg 2000, 232, 619-626.

108. Sunkara, T.; Caughey, M.E.; Rawla, P.; Yarlagadda, K.S.; Gaduputi, V. Severe Acute Pancreatitis as an Index Clinical Manifestation of Parathyroid Adenoma. Cureus 2018, 10, e2445, doi:10.7759/cureus.2445.

109. Kim, K.O.; Kim, T.N. Acute pancreatic pseudocyst: incidence, risk factors, and clinical outcomes. Pancreas 2012, 41, 577-581, doi:10.1097/MPA.0b013e3182374def.

110. Byrne, M.F.; Mitchell, R.M.; Baillie, J. Pancreatic Pseudocysts. Current treatment options in gastroenterology 2002, 5, 331-338.

111. Srikanth, G.; Sikora, S.S.; Baijal, S.S.; Ayyagiri, A.; Kumar, A.; Saxena, R.; Kapoor, V.K. Pancreatic abscess: 10 years experience. ANZ journal of surgery 2002, 72, 881-886.

112. Evans, R.P.; Mourad, M.M.; Pall, G.; Fisher, S.G.; Bramhall, S.R. Pancreatitis: Preventing catastrophic haemorrhage. World J Gastroenterol 2017, 23, 5460-5468, doi:10.3748/wjg.v23.i30.5460.

113. Flati, G.; Andren-Sandberg, A.; La Pinta, M.; Porowska, B.; Carboni, M. Potentially fatal bleeding in acute pancreatitis: pathophysiology, prevention, and treatment. Pancreas 2003, 26, 8-14.

114. Rawla, P.; Sunkara, T.; Gaduputi, V. Epidemiology of Pancreatic Cancer: Global Trends, Etiology and Risk Factors. World journal of oncology 2019, 10, 10-27, doi:10.14740/wjon1166.

115. Navadgi, S.; Pandanaboyana, S.; Windsor, J.A. Surgery for Acute Pancreatitis. The Indian journal of surgery 2015, 77, 446-452, doi:10.1007/s12262-015-1357-x.

116. Werner, J.; Feuerbach, S.; Uhl, W.; Buchler, M.W. Management of acute pancreatitis: from surgery to interventional intensive care. Gut 2005, 54, 426-436, doi:10.1136/gut.2003.035907.

117. Busquets, J.; Fabregat, J.; Pelaez, N.; Millan, M.; Secanella, L.; Garcia-Borobia, F.; Masuet, C.; MartinezGarcia, L.; Lopez-Borao, J.; Valls, C., et al. Factors Influencing Mortality in Patients Undergoing Surgery for Acute Pancreatitis. Pancreas 2013, 42, 285-292, doi:10.1097/mpa.0b013e318264664d.

118. Nordback, I.; Pelli, H.; Lappalainen-Lehto, R.; Järvinen, S.; Räty, S.; Sand, J. The Recurrence of Acute Alcohol-Associated Pancreatitis Can Be Reduced: A Randomized Controlled Trial. Gastroenterology 2009, 136, 848-855, doi:10.1053/j.gastro.2008.11.044.

119. Ahmed Ali, U.; Issa, Y.; Hagenaars, J.C.; Bakker, O.J.; van Goor, H.; Nieuwenhuijs, V.B.; Bollen, T.L.; van Ramshorst, B.; Witteman, B.J.; Brink, M.A., et al. Risk of Recurrent Pancreatitis and Progression to Chronic 
Pancreatitis After a First Episode of Acute Pancreatitis. Clinical gastroenterology and hepatology : the official clinical practice journal of the American Gastroenterological Association 2016, 14, 738-746, doi:10.1016/j.cgh.2015.12.040.

120. Guda, N.M.; Romagnuolo, J.; Freeman, M.L. Recurrent and relapsing pancreatitis. Curr Gastroenterol Rep 2011, 13, 140-149, doi:10.1007/s11894-011-0176-x. 